\title{
No association between a promoter NOS1 polymorphism (rs41279104) and Infantile Hypertrophic Pyloric Stenosis
}

\author{
Kristina Lagerstedt-Robinson ${ }^{1}$, Anna Svenningsson ${ }^{1,2}$ and Agneta Nordenskjöld ${ }^{2,3}$
}

Infantile hypertrophic pyloric stenosis (IHPS) is a condition affecting infants in the first few months of life. The condition is manifested by persistent vomiting and is caused by a hypertrophied muscle obstructing the gastric outlet. The condition is treated by pyloromyotomy. The incidence is $1-8 / 1000$ births and varies among different populations. The etiology of IHPS is unknown, but both genetic and environmental factors are thought to contribute to the disease. Genetic linkage analysis has so far localized five loci that could harbor genes contributing to IHPS. The only gene implicated in IHPS is the nitric oxide synthase gene (NOS1), in which a single nucleotide polymorphism (rs41279104) in the promoter region has been associated with the disease in 16 patients. In this study, we examined an association of this SNP in 54 familial and 28 sporadic cases with IHPS, and compared the results with normal controls using univariate and multiple logistic regression analysis. We could not confirm any association between the analyzed SNP and infantile hypertrophic pyloric stenosis.

Journal of Human Genetics (2009) 54, 706-708; doi:10.1038/jhg.2009.101; published online 23 October 2009

Keywords: association; infantile hypertrophic pyloric stenosis; nitric oxide synthase; single nucleotide polymorphism

\section{INTRODUCTION}

Infantile hypertrophic pyloric stenosis (IHPS, (OMIM no. 179010)) is a condition affecting infants in their first few months of life. IHPS is caused by hypertrophy of the circular muscle layer of the pylorus, leading to persistent vomiting typically at 3 weeks of age. The incidence is $1-8 / 1000$ in Caucasians and incidence varies greatly among other populations. ${ }^{1}$ Males are more commonly affected than females, with reported ratios of $4: 1 .^{2}$

The causes of IHPS are multifactorial, in which both environmental factors and hereditary factors are believed to contribute. Possible etiological factors include deficiency of nitric oxide synthase containing neurons, abnormal myenteric plexus innervation, infantile hypergastrinemia and recently exposure to macrolide antibiotics. ${ }^{3-5}$ Genetic linkage analyses of familial cases with IHPS have suggested five loci that could harbor genes contributing to IHPS; $12 \mathrm{q} 24.2-\mathrm{q} 24.31,{ }^{6}$ 16p13-p12, 11q14-q22, ${ }^{8} \mathrm{Xq} 23^{8}$ and $16 \mathrm{q} 24 .{ }^{9}$ The only gene, so far, that has been implicated with the development of IHPS is the NOS1 gene. ${ }^{10}$ NOS1 is located on chromosome $12 \mathrm{q}$ and encodes neuronal nitric oxide synthase.

Nitric oxide is a major inhibitory nonadrenergic, noncholinergic neurotransmitter in the GI tract, causing relaxation of smooth muscle of the mammalian digestive tract upon its release. This raises the possibility that pylorospasm could be caused by a defect in nitric oxide production. Neuronal nitric oxide synthase (NOS1) is the enzyme that catalyses the formation of nitric oxide in neurons in both the central and the peripheral nervous system. The neuronal isoform (nNOS) of the enzyme is one of three described isoforms, the others being inducible NOS and endothelial NOS. nNOS has been described as the main source for production of NO in the gut. ${ }^{11}$ Impairment of nNOS synthesis has been implicated in IHPS ${ }^{10}$ and also in other manifestations such as achalasia, diabetic gastroparesis and Hirschsprung disease, reviewed in reference. ${ }^{12}$

Also, knockout mice with targeted disruption of the neuronal Nos (Nos1) gene developed enlarged stomach with hypertrophy of the pyloric sphincter and the circular muscle layer. ${ }^{13}$

A genetic alteration in the human NOS1 exon 1c regulatory region influences expression of the NOS1 gene. ${ }^{14} \mathrm{~A}$ reporter gene assay has shown that an A-allele in the exon 1c promoter SNP c.-84G $>$ A (single nucleotide polymorphism, also known as rs41279104) located in a putative regulatory region has an approximately $30 \%$ reduced expression of NOS1. Furthermore, this allele has been shown to be a risk factor for IHPS. ${ }^{14}$

This prompted us to examine the association between the c.-84G $>$ A SNP in the NOS1 gene and IHPS, in a large cohort of familial and sporadic cases of patients with IHPS.

${ }^{1}$ Department of Molecular Medicine and Surgery, Karolinska Institutet, Stockholm, Sweden; ${ }^{2}$ Pediatric Surgery, Astrid Lindgren Children Hospital, Karolinska University Hospital, Stockholm, Sweden and ${ }^{3}$ Department of Woman and Child Health, Karolinska Institutet, Stockholm, Sweden

Correspondence: Dr K Lagerstedt-Robinson, Department of Molecular Medicine and Surgery, Karolinska Institutet, Karolinska University Hospital, L5:03, Stockholm SE-17176, Sweden.

E-mail: kristina.lagerstedt@ki.se

Received 8 July 2009; revised 21 September 2009; accepted 26 September 2009; published online 23 October 2009 


\section{MATERIALS AND METHODS}

Patients with IHPS were identified from Swedish Paediatric Surgery Clinics in Sweden (Stockholm, Gothenburg, Uppsala and Lund). All patients were clinically diagnosed with isolated IHPS and underwent surgical treatment. Patients affected with IHPS as part of another genetic syndrome were not included in this study. The patient's parents were asked for additional familial cases and then interviewed over the telephone to construct a pedigree. Skin biopsies or blood were sampled after informed consent. In total, the patient group consisted of 82 patients; 54 of them were familial cases (two or more affected individuals in the same family) and 28 were sporadic (no known family history). The sporadic cases were all male patients. In the familial cases 18 were female and 36 were male patients. In the case of a familial inheritance, one individual per family was included in the study. The control group consisted of a total of 80 anonymous blood donors ( 37 females and 43 males). The study was approved by the Research Ethics Committee of Karolinska Institutet.

Genomic DNA was prepared from fibroblasts or peripheral blood using a standard protocol using phenol and chloroform. To determine the sex of the individuals in the control group the $S R Y$ gene was amplified from genomic DNA using PCR. Primers and amplification conditions are available on request.

The promotor sequence of exon 1c of the NOS1 gene was amplified using PCR with the primers 'NOS1 exlc fwd' (5'-AGGTCAGAGCCTGGGGAAGC-3') and 'NOS1 ex1c rev' (5'-CTCCTGCCTGGGGAGCTGAG-3'). The PCRs were performed using $1 \times$ PCRAmpBuffer (Invitrogen, Life Technologies Corporation, CA, USA), $1.5 \mathrm{~mm} \mathrm{MgSO} 4$ (Invitrogen), $2 \times$ PCR Enhancer solution (Invitrogen), $1.25 \mathrm{U}$ Taq DNA Polymerase (Invitrogen), $0.2 \mathrm{~mm}$ dNTP (Larova Biochemie GmbH, Teltow, Germany), $2 \mu \mathrm{m}$ of each primer (Thermo Electron Corporation, Thermo Fisher Scientific GmbH, Ulm, Germany) and approximately $100 \mathrm{ng}$ of genomic DNA in a total volume of $25 \mu \mathrm{l}$. PCR amplification was performed at $95^{\circ} \mathrm{C}$ for $2 \mathrm{~min}$ followed by 35 cycles at $95^{\circ} \mathrm{C}$ for $45 \mathrm{~s}, 63^{\circ} \mathrm{C}$ for $30 \mathrm{~s}, 68^{\circ} \mathrm{C}$ for $1 \mathrm{~min}$ and a final extension step at $68^{\circ} \mathrm{C}$ for $4 \mathrm{~min}$. All amplifications were performed in a PTC100 (MJ Research/Bio-Rad Laboratories Inc, Sundbyberg, Sweden).

The PCR products were purified from unused dNTP and primers using ExoSap (USB Corporation GmbH, Staufen, Germany) according to the manufacturer's instructions. DNA sequence reactions were performed using BigDye Terminator v1.1 Cycle Sequencing kit (Applied Biosystems, Foster City, CA, USA) according to the manufacturer's recommendations and analyzed on an ABI 3100 (Applied Biosystems). Chromatograms were generated using Sequencing analysis (Applied Biosystems).

Data were analyzed using univariate and multiple logistic regression analysis, using SAS System 9.1 (SAS Institute Inc., Cary, NC, USA). An exact logistic regression analysis was performed when the cells in the cross-tabulation of the outcome variable status and the independent variables genotype and gender were less than 5 . When comparing familial and sporadic cases to the controls regarding genotype (G/G and A-carriers) a generalized logit model was used and optimization was performed using Fisher's scoring. The same parameters were used when comparing cases with controls vs. genotype (G/G and A-carriers) and also when searching for possible interactions together with male or female sex in the familial cases. Results were presented as odds ratios with $95 \%$ confidence intervals. $P<0.05$ was considered statistically significant.

\section{RESULTS}

Using DNA sequencing analysis the frequency of the different genotypes $(\mathrm{G} / \mathrm{G}, \mathrm{A} / \mathrm{G}$ or $\mathrm{A} / \mathrm{A})$ of an SNP located in the promoter region of the NOS1 gene were determined for each individual. Frequencies of the genotypes were determined in familial cases (54 individuals) and sporadic cases with IHPS (28 individuals) and compared with the frequencies observed in a control group (80 individuals).

No association between carriers of the A-allele in the NOS1 gene and IHPS was observed (data shown in Table 1). Comparing homozygous G-carriers with A-carriers in the familial cases $(\mathrm{OR}=0.43 ; 95 \%$ CI: $0.17-1.05 ; P=0.06)$ and in the sporadic cases $(\mathrm{OR}=0.53 ; 95 \% \mathrm{CI}$ : $0.18-1.59 ; P=0.26)$ no statistical difference could be observed. Also when comparing all cases, familial and sporadic together in a group, with controls no significant association was detected $(\mathrm{OR}=0.47 ; 95 \%$ CI: $0.21-1.00 ; P=0.05)$ regarding A-carriers compared with $G / G$ carriers.

Finally, taking genotype (homozygous G-carriers or A-carriers), sex (male or female) and familial inheritance together no interaction could be detected ( $\mathrm{OR}=1.10 ; 95 \% \mathrm{CI}: 0.13-10.89 ; P=1.00)$ using multiple logistic regression analysis.

\section{DISCUSSION}

Many previous publications have suggested that nNOS expression have a role in pathogenesis of IHPS. Several studies have shown that IHPS patients have a reduced expression of nNOS in the investigated area of interest. ${ }^{10,15,16}$ Previous results have also shown that in the presence of the c.-84A SNP a 30\% reduction of NOS1C expression can be seen using TaqMan expression analysis. ${ }^{14}$

In this study, we have analyzed a large group of patients with IHPS with regard to a single SNP, in which, previously the A-allele has been associated with IHPS in a small subset of cases. ${ }^{14}$ Our patient material consisted of both familial and sporadic cases affected with isolated IHPS. Genetic and statistical analysis of the results regarding a possible association of the c.-84 SNP in the promoter region of the investigated gene, sex of affected familial and/or sporadic patient failed to find any significant correlation. In contrast, our findings show that the G-allele of this SNP was more common in the patients compared with the controls (Table 1), although this finding was border-line significant $(P=0.05)$. This is in opposition to previously published results, and suggests that some other mechanisms than this SNP are involved. However, one must consider that the NOS promoter diversity is one of the most complicated described so far, it is not unlikely that expression of NOS involves many different factors.

In this study, we have analyzed in total 82 patients operated for IHPS and therefore have more statistical power compared with the 16 cases that were investigated in an earlier study. Another discrepancy between the two studies is the allele frequencies in the control groups. In our control group the frequency of carriers in the A-allele is $29 \%$, but in the publication by Saur et al. ${ }^{14}$ this frequency was only $17 \%$. The reason for this is so far unknown, but the genetic difference between the population in Sweden compared with Germany probably cannot explain the whole difference. The difference between the two studies could also be because of different sample sizes.

IHPS is more common in male compared with female patients with a reported sex ratio of 4:1. ${ }^{2}$ In our collection of sporadic cases we have

Table 1 Genotype frequencies of SNP rs41279104 seen in NOS1 promoter in patients (familial and sporadic cases) compared with controls

\begin{tabular}{|c|c|c|c|c|}
\hline Genotype (rs41279104) & Patients familial cases $(n=54)$ & Sporadic cases $(n=28)$ & Patients total $(\mathrm{n}=82)$ & Controls $(n=80)$ \\
\hline $\mathrm{G} / \mathrm{G}$ & $46(85 \%)$ & $23(82 \%)$ & $69(84 \%)$ & $57(71 \%)$ \\
\hline$A / G$ & 7 (13\%) & $5(18 \%)$ & $12(15 \%)$ & $23(29 \%)$ \\
\hline$A / A$ & $1(2 \%)$ & 0 & $1(1 \%)$ & 0 \\
\hline
\end{tabular}


a sex ratio of 13:1 (data not shown). The reason for this discrepancy may be a bias on our collected material, in which familial cases were more predominant, and therefore not so successful in collecting all sporadic cases. It could also reflect the fact that affected female patients might be clustered in families. In our study, all our 28 analyzed sporadic cases were male patients. The reason for this is thought to be the sampling bias described above.

So far the NOS1 gene has been the best candidate gene for IHPS. The expression of NOS1 has been shown to be reduced in patients with the disorder. In addition linkage mapping has proven linkage in the region, in which NOS1 is located (Svenningsson et al., manuscript in preparation), suggesting that the NOS1 gene is the best candidate gene for IHPS, so far. Further studies are recommended to explain the reduced expression of the NOS1 gene in IHPS patients. Genetic screening of the whole NOS1 gene is recommended, to investigate other possible genetic alterations in NOS1 that could be of importance for the development of IHPS.

\section{CONFLICT OF INTEREST}

The authors declare that they have no conflict of interest.

\section{ACKNOWLEDGEMENTS}

This work was supported by grants from the Swedish Research Council, HRH Crown Princess Lovisa Foundation, Karolinska Institutet, Foundation Frimurare Barnhuset and the Sven Jerring foundation. We thank Malin Smith for technical assistance.

1 Schechter, R., Torfs, C. P. \& Bateson, T. F. The epidemiology of infantile hypertrophic pyloric stenosis. Paediatr Perinat Epidemiol 11, 407-427 (1997).

2 Carter, C. O. The inheritance of congenital pyloric stenosis. Br Med Bull 17, 251-254 (1961).
3 Honein, M. A., Paulozzi, L. J., Himelright, I. M., Lee, B., Cragan, J. D., Patterson, L. et al. Infantile hypertrophic pyloric stenosis after pertussis prophylaxis with erythromycin: a case review and cohort study. Lancet 354, 2101-2105 (1999).

4 Mahon, B. E., Rosenman, M. B. \& Kleiman, M. B. Maternal and infant use of erythromycin and other macrolide antibiotics as risk factors for infantile hypertrophic pyloric stenosis. J Pediatr 139, 380-384 (2001).

5 Cooper, W. O., Griffin, M. R., Arbogast, P., Hickson, G. B., Gautam, S. \& Ray, W. A. Very early exposure to erythromycin and infantile hypertrophic pyloric stenosis. Arch Pediatr Adolesc Med 156, 647-650 (2002)

6 Chung, E., Curtis, D., Chen, G., Marsden, P. A., Twells, R., Xu, W. et al. Genetic evidence for the neuronal nitric oxide synthase gene (NOS1) as a susceptibility locus for infantile pyloric stenosis. Am J Hum Genet 58, 363-370 (1996).

7 Capon, F., Reece, A., Ravindrarajah, R. \& Chung, E. Linkage of monogenic infantile hypertrophic pyloric stenosis to chromosome 16p12-p13 and evidence for genetic heterogeneity. Am J Hum Genet 79, 378-382 (2006).

8 Everett, K. V., Chioza, B. A., Georgoula, C., Reece, A., Capon, F., Parker, K. A. et al. Genome-wide high-density SNP-based linkage analysis of infantile hypertrophic pyloric stenosis identifies loci on chromosomes 11q14-q22 and Xq23. Am J Hum Genet 82, 756-762 (2008)

9 Everett, K. V., Capon, F., Georgoula, C., Chioza, B. A., Reece, A., Jaswon, M. et al. Linkage of monogenic infantile hypertrophic pyloric stenosis to chromosome 16q24. Eur J Hum Genet 16, 1151-1154 (2008).

10 Kusafuka, T. \& Puri, P. Altered messenger RNA expression of the neuronal nitric oxide synthase gene in infantile hypertrophic pyloric stenosis. Pediatr Surg Int 12, 576-579 (1997).

11 Saur, D., Paehge, H., Schusdziarra, V. \& Allescher, H. D. Distinct expression of splice variants of neuronal nitric oxide synthase in the human gastrointestinal tract. Gastroenterology 118, 849-858 (2000).

12 Takahashi, T. Pathophysiological significance of neuronal nitric oxide synthase in the gastrointestinal tract. J Gastroenterol 38, 421-430 (2003).

13 Huang, P. L., Dawson, T. M., Bredt, D. S., Snyder, S. H. \& Fishman, M. C. Targeted disruption of the neuronal nitric oxide synthase gene. Cell 75, 1273-1286 (1993).

14 Saur, D., Vanderwinden, J. M., Seidler, B., Schmid, R. M., De Laet, M. H. \& Allescher, H. D. Single-nucleotide promoter polymorphism alters transcription of neuronal nitric oxide synthase exon 1c in infantile hypertrophic pyloric stenosis. Proc Natl Acad Sci USA 101, 1662-1667 (2004).

15 Gentile, C., Romeo, C., Impellizzeri, P., Turiaco, N., Esposito, M., Di Mauro, D. et al. A possible role of the plasmalemmal cytoskeleton, nitric oxide synthase, and innervation in infantile hypertrophic pyloric stenosis. A confocal laser scanning microscopic study. Pediatr Surg Int 14, 45-50 (1998).

16 Huang, L. T., Tiao, M. M., Lee, S. Y., Hsieh, C. S. \& Lin, J. W. Low plasma nitrite in infantile hypertrophic pyloric stenosis patients. Dig Dis Sci 51, 869-872 (2006). 